\title{
Effect of Isoproterenol on Renal Uric Acid Excretion in Rats
}

\author{
Haruko SUGINO and Hideyo SHIMADA \\ Department of Clinical Pharmacology. School of Pharmaceutical Sciences, \\ Kitasato University, Shirokane 5-9-1. Minato-ku, Tokyo 108, Japan
}

Accepted July 27, 1987

\begin{abstract}
Effect of isoproterenol on renal uric acid excretion was examined in oxonate-loaded and oxonate-nonloaded rats, using a clearance technique. Oxonate loading was performed by continuous infusion into the femoral vein. Isoproterenol (50 $\mathrm{kg} / \mathrm{kg}$, i.v.) induced antidiuresis and hyperuricemia accompanied with decreases in urinary excretion rate of uric acid, uric acid clearance, inulin clearance, and uric acid clearance/inulin clearance ratio in oxonate-nonloaded rats. These effects of isoproterenol were inhibited by propranolol ( $1 \mathrm{mg} / \mathrm{kg}$, i.v.). Very similar results were obtained in oxonate-loaded rats: i.e., systemic blood pressure decreased synchronously with uric acid excretion by isoproterenol. In contrast, phenylephrine $(100 \mu \mathrm{g} / \mathrm{kg}$, i.v.) induced hypertensive, diuretic and slightly uricosuric results. In allopurinol-pretreated and oxonate-loaded rats, isoproterenol also decreased renal uric acid excretion and showed a less potent hyperuricemic effect than that observed in the animals not pretreated with allopurinol. In addition, in rats with ligated renal vessels, the hyperuricemic effect of isoproterenol was completely inhibited by allopurinol. These results suggest that isoproterenol-induced hyperuricemia is related not only to the stimulation of uric acid production, but also to the depression of renal uric acid excretion.
\end{abstract}

Catecholamines such as epinephrine and isoproterenol are known to induce hyperuricemia in rats $(1,2)$. Regulatory mechanisms of plasma uric acid level involve alterations of both uric acid production and uric acid excretion (3). Yonetani et al. (4) reported that hyperuricemia induced by epinephrine and isoproterenol in rats is caused by the stimulation of uric acid production. However, little information on the relationship between the hyperuricemic and renal actions of isoproterenol is available. Using a clearance technique in rats loaded with potassium oxonate, a uricase inhibitor (5). the present study was designed to determine whether or not the mechanism of hyperuricemic action of isoproterenol involves alteration of renal uric acid excretion.

\section{Materials and Methods}

Animals and treatments: Male Wistar rats, 7 weeks of age, were anesthetized with urethane $(1.2 \mathrm{~g} / \mathrm{kg}$, s.c. $)$. The left femoral vein was cannulated with a polyethylene tube (PE-10) for infusion of loading solution. The loading solutions were as follows: Fluid I contained 4\% mannitol, 1.5\% inulin and $0.85 \% \mathrm{NaCl}$. Fluid $1 /$ consisted of fluid I with $0.1 \%$ oxonate and $0.03 \% \quad \mathrm{NaHCO}_{3}$. Fluid III consisted of fluid I with $1.5 \mathrm{mM}$ uric acid. $0.1 \%$ oxonate and $0.055 \% \quad \mathrm{NaHCO}_{3}$. Uric acid was added to obtain a higher plasma level. The loading solution was infused at a rate of $3.2 \mathrm{ml} / \mathrm{hr}$. The urinary bladder was catheterized with Tygon tubing for collection of urine. After a $60 \mathrm{~min}$ equilibrium period, urine collection, blood collection and drug administration were done as follows: $10 \mathrm{~min}$ urine was collected continuously. Blood $(0.37 \mathrm{ml}$, each) was taken from the jugular vein with a heparinized syringe $10 \mathrm{~min}$ before, and 10 and $30 \mathrm{~min}$ after the administration of test drugs or saline as control (Fig. 1). After centrifugation of the blood, the plasma sample was obtained.

Uric acid concentrations in plasma and urine were measured by the phosphotungstate colorimetric assay using a Uric Acid-Test 


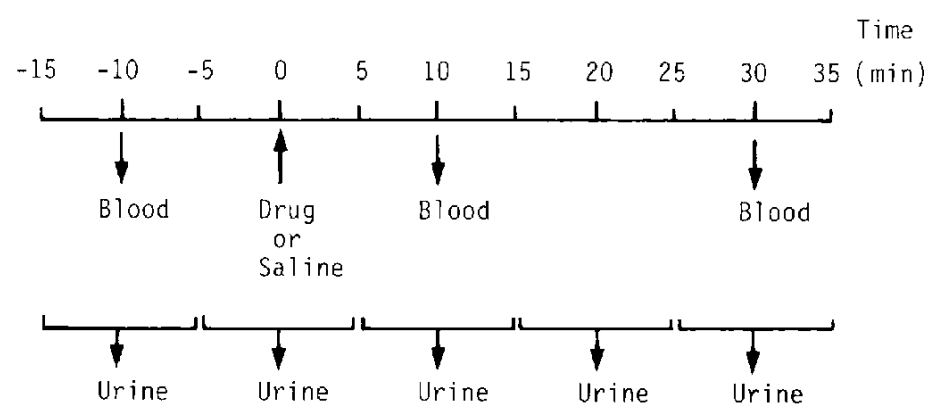

Fig. 1. Experimental protocol for collection of urine and blood and administration of drugs.

Wako kit (Wako). Inulin concentrations in plasma and urine were determined by the fluorometric method of Vurek and Pegram (6).

Systemic blood pressure was measured with a pressure transducer (Nihon Kohden, MPU-0.5) from a polyethylene tube (PE-50) inserted into the right femoral artery.

Rats with ligated renal vessels were prepared as follows: The arteries and veins of both kidneys were ligated at the neck of the kidney.

Chemicals: The following drugs were used: isoproterenol hydrochloride (Nikken Kagaku). phenylephrine hydrochloride (Tokyo Kasei), dl-propranolol hydrochloride and allopurinol (Sigma), mannitol and inulin (Nakarai). potassium oxonate (Calbiochem-Behring). Other chemicals were of reagent grade. Phenylephrine and propranolol were dissolved in saline. Isoproterenol was diluted with saline. Allopurinol was suspended in $0.2 \%$ sodium carboxymethyl cellulose.

Statistical analysis: The data are expressed as the mean \pm S.D. and statistically analyzed by Student's t-test.

\section{Results}

Effect of isoproterenol on renal uric acid excretion in oxonate-nonloaded rats: Fluid I was used as the loading solution in this experiment.

Urine volume $\left(U_{V_{01}}\right)$, urinary excretion rate of uric acid $\left(U_{U A}\right)$. plasma concentration of uric acid $\left(P_{U_{A}}\right)$. uric acid clearance $\left(C_{V_{A}}\right)$. inulin clearance $\left(C_{I n}\right)$ and uric acid clearance/ inulin clearance ratio $\left(\mathrm{C}_{\mathrm{UA}} / \mathrm{C}_{\mathrm{In}_{n}}\right)$ before drug administration were $26.5 \pm 2.7 \mu / / \mathrm{min}, 4.35 \pm$ $1.40 \% \mathrm{~g} / \mathrm{min}, 0.55 \pm 0.10 \mathrm{mg} / \mathrm{dl}, 0.769 \pm 0.322$ $\mathrm{ml} / \mathrm{min}, 2.09 \pm 0.46 \mathrm{ml} / \mathrm{min}$ and $0.380 \pm 0.163$, respectively. In the control group, saline had no effect on any of the parameters. Isoproterenol $(50 \mu \mathrm{g} / \mathrm{kg}$, i.v.) showed an antidiuretic effect immediately after the administration. and it also caused transient decreases in $U_{U A}$. $C_{C_{A}}, C_{I n}$ and $C_{U A} / C_{I n}$; the decreases in $U_{U A}$. $C_{P A}$ and $C_{U A} / C_{I n}$ were followed by increases. $P_{C A}$ clearly increased (about 2 -fold). Propranolol (1 mg/kg, i.v.) administered $1 \mathrm{~min}$ before isoproterenol completely blocked the effect of isoproterenol. Propranolol alone or propranolol plus isoproterenol induced mild diuresis (Fig. 2).

Effects of isoproterenol and phenylephrine on renal uric acid excretion and blood pressure in oxonate-loaded rats: Fluid II was infused in this experiment.

$U_{\text {vol }}, U_{U A}, P_{\text {CA }}, C_{V_{A}}, C_{I n}$ and $C_{U A} / C_{\text {In }}$ before drug administration were $26.9 \pm 4.8 \mu \mathrm{l} /$ $\mathrm{min}, 31.7 \pm 4.9 \mu \mathrm{g} / \mathrm{min}, 1.95 \pm 0.35 \mathrm{mg} / \mathrm{dl}$. $1.61 \pm 0.28 \mathrm{ml} / \mathrm{min}, 2.17 \pm 0.39 \mathrm{ml} / \mathrm{min}$ and $0.784 \pm 0.141$, respectively: oxonate produced higher levels of $U_{U_{A}}, P_{C_{A}}, C_{U_{A}}$ and $C_{U A} / C_{I n}$ than those obtained in oxonate-nonloaded rats, but it did not affect $U_{v o l}$ and $C_{I n}$. The antidiuretic effect of isoproterenol $(50 \mu \mathrm{g} / \mathrm{kg}$, i.v.) in oxonate-loaded rats was almost the same in potency as in oxonate-nonloaded rats. In addition, responses in the other parameters to isoproterenol in oxonateloaded rats and oxonate-nonloaded rats were similar except that, in oxonate-loaded rats, increases in $\mathrm{C}_{\mathrm{UA}}$ and $\mathrm{C}_{\mathrm{UA}} / \mathrm{C}_{\mathrm{In}}$ at $30 \mathrm{~min}$ after the administration were not observed. In contrast, phenylephrine (100 $/ \mathrm{g} / \mathrm{kg}$, i.v.) was diuretic and transiently increased $\mathrm{U}_{\mathrm{U}_{A}}$. but did not affect $P_{C^{-A}}, C_{T_{A}}, C_{I_{n}}$ and $C_{C_{A}} / C_{I^{n}}$ (Fig. 3).

The effects of isoproterenol and phenyl- 
ephrine on systemic (mean) blood pressure were evaluated simultaneously with clearance studies. In the control group, blood pressure did not change during the experimental period. Isoproterenol, however, lowered blood

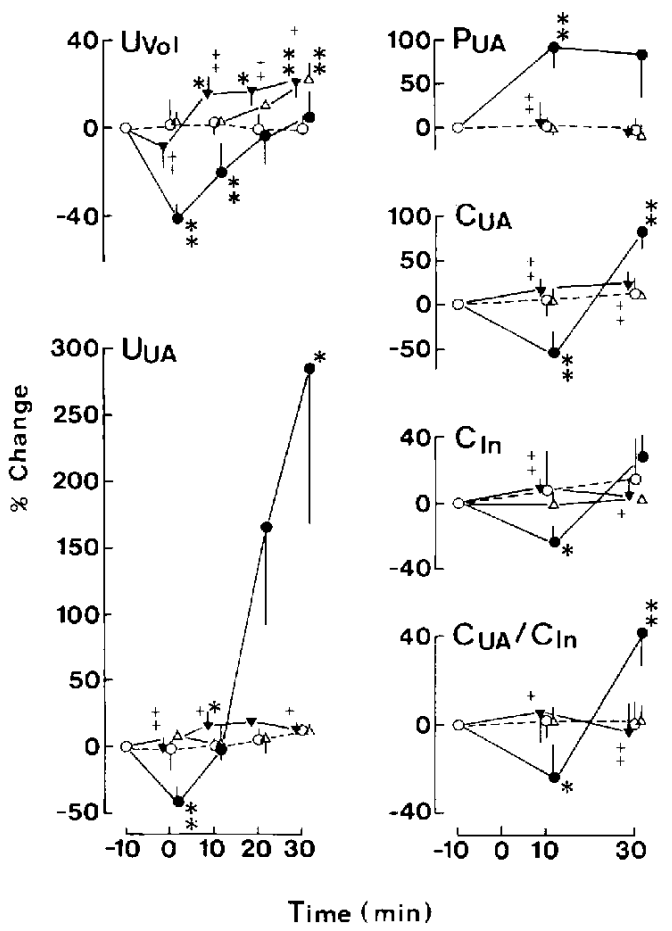

Fig. 2. Effects of isoproterenol and propranolol on uric acid excretion in rats. Isoproterenol was administered at time 0 . Propranolol was administered $1 \mathrm{~min}$ before isoproterenol. Results are expressed as \% changes from the values before drug administration. Each point represents the meantS.D. $(N=5)$. ${ }^{*} P<0.05,{ }^{*} P<0.01$, significantly different from the contral. $+P<0.05,++P<0.01$, significantly different from isoproterenol. $\bigcirc$ : control. $O$ : isoproterenol $(50$ $\mu \mathrm{g} / \mathrm{kg}, \mathrm{i} . \mathrm{v}$ ). $\Delta$ : propranolol (1 $\mathrm{mg} / \mathrm{kg}, \mathrm{i} . \mathrm{v}$.$) .$ propranolol+isoproterenol. pressure and at the same time decreased $U_{\text {Vol }}$ and $U_{U_{A}}$. Phenylephrine raised blood pressure at the same time increasing $U_{U_{A}}$ (Table 1).

Effect of isoproterenol on renal uric acid excretion in allopurinol-pretreated and oxonate-loaded rats: The animals were pretreated with allopurinol (100 mg/kg, p.o.) $2.5 \mathrm{hr}$ before isoproterenol administration.

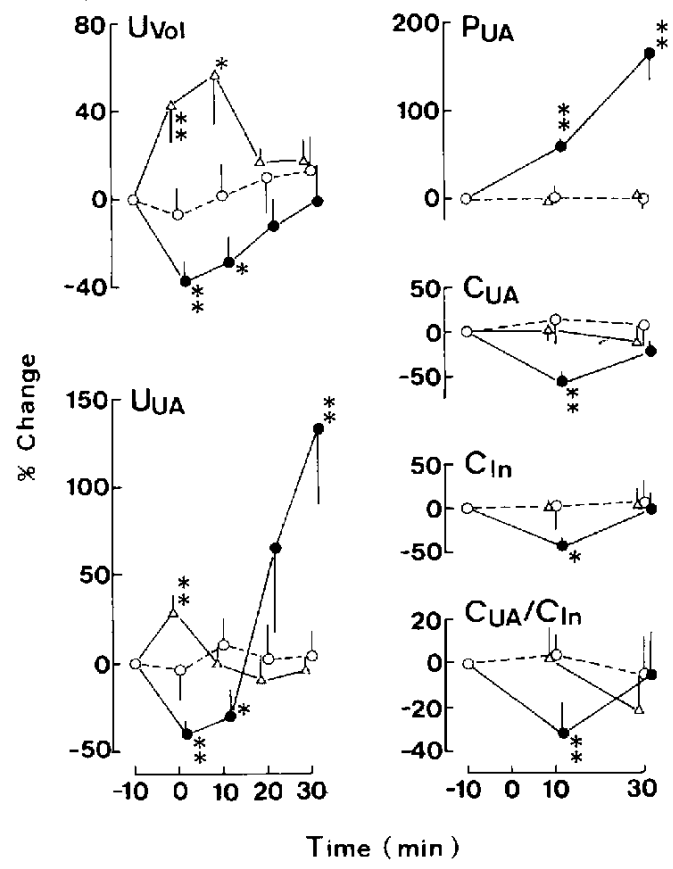

Fig. 3. Effects of isoproterenol and phenylephrine on renal uric acid excretion in oxonate-loaded rats. Each drug was administered at time 0 . Results are expressed as \% changes from the values before drug administration. Each point represents the mean $t S . D$. $(N=5-6) .{ }^{*} P<0.05,{ }^{*} P<0.01$, significantly different from the control. $O$ : control, $\mathrm{O}$ isoproterenol $(50 \mu \mathrm{g})$ $\mathrm{kg}$, i.v.), $\triangle$ : phenylephrine (100 $\mu \mathrm{g} / \mathrm{kg}$, i.v.).

Table 1. Effects of isoproterenol and phenylephrine on mean blood pressure in oxonate-loaded rats

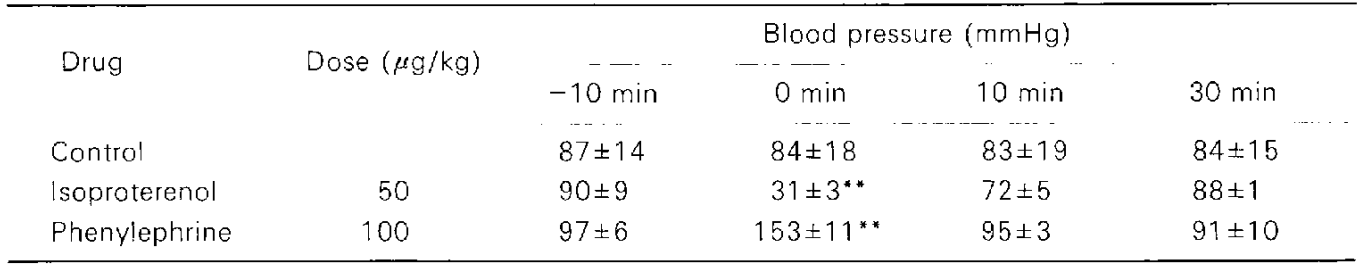

Each drug was administered intravenously at time 0 . Data represent the mean $\pm S . D . \quad(N=4-6)$. ${ }^{*} P<0.01$, significantly different from the control. 
then infused with fluid $\mathbf{I I}$.

$\mathrm{U}_{\mathrm{Vo} 1}, \mathrm{U}_{\mathrm{UA}}, \mathrm{P}_{\mathrm{UA}}, \mathrm{C}_{\mathrm{UA}}, \mathrm{C}_{\mathrm{In}}$ and $\mathrm{C}_{\mathrm{UA}} / \mathrm{C}_{\mathrm{In}}$ before isoproterenol were $32.4 \pm 9.3 \mu \mathrm{l} / \mathrm{min}$. $27.5 \pm 7.2 \mu \mathrm{g} / \mathrm{min}, 1.53 \pm 0.36 \mathrm{mg} / \mathrm{dl}, 1.81 \pm$ $0.27 \mathrm{ml} / \mathrm{min}, 2.13 \pm 0.36 \mathrm{ml} / \mathrm{min}$ and $0.847 \pm$ 0.155 , respectively. In these allopurinolpretreated and oxonate-loaded rats, isoproterenol $(50 \mu \mathrm{g} / \mathrm{kg}$, i.v.) induced antidiuresis and caused a decrease followed by an increase in $U_{U A}$, while the increase in $U_{\mathrm{CA}_{1}}$ was less than that observed in the animals not

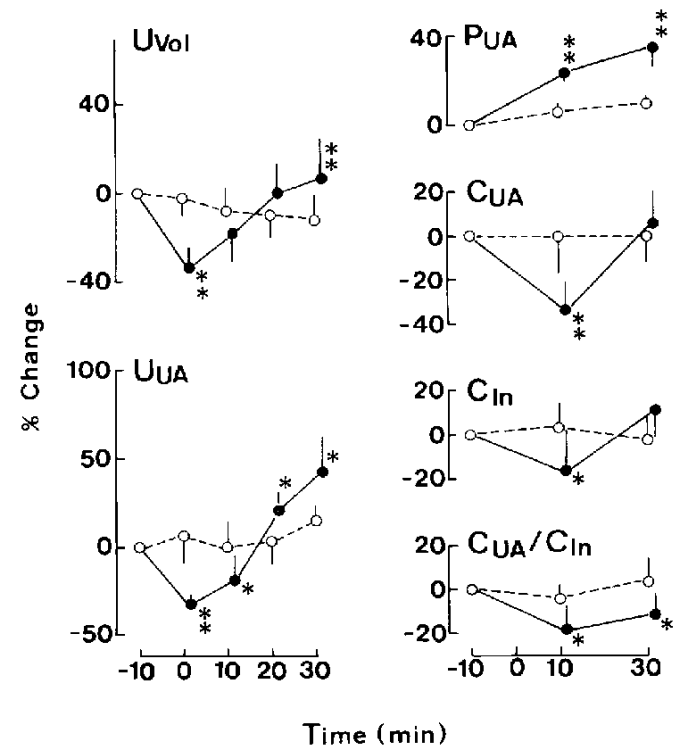

Fig. 4. Effect of isoproterenol on rena! uric acid excretion in allopurinol-pretreated and oxonateloaded rats. I soproterenol was administered at time 0 . Results are expressed as \% changes from the values before drug administration. Each point represents the meantS.D. $\quad(N=6) . \quad * P<0.05, \quad * P<0.01$, significantly different from the contro!. $O$ : control. isoproterenol $(50 \mu \mathrm{J} / \mathrm{kg}, \mathrm{i} . \mathrm{v})$. pretreated with allopurinol. $P_{\mathbf{U A}_{A}}$ increased slightly and gradually: the increase was statistically significant $(P<0.01) . \quad C_{U N}, C_{I n}$ and $\mathrm{C}_{\mathrm{UA}} / \mathrm{C}_{\mathrm{In}}$ decreased (Fig. 4).

Effect of isoproterenol on plasma uric acid in rats with ligated renal vessels: Ligation of renal vessels was performed to eliminate the influence of renal functions. After this operation, $P_{\text {LA }}$ gradually increased. Isoproterenol (50 $\mu \mathrm{g} / \mathrm{kg}$. i. . ) potentiated the increase in $P_{\mathrm{VA}}$. However, the hyperuricemic effect of isoproterenol was lost in rats pretreated with allopurinol (100 mg/kg. p.o.) (Table 2).

\section{Discussion}

Because most uric acid is rapidly converted into allantoin by uricase in rats, treatment with oxonate is a very useful method for studying the regulatory mechanisms of plasma uric acid level in rats. Yonetani and Iwaki (7) reported the usefulness of rats intraperitoneally treated with oxonate for evaluating renal effects of drugs. De Rougemont et al. (8) stated that the infusion of oxonate did not influence the water and urate transport. Accordingly, the present experiments were carried out employing a clearance technique using rats infused continuously with oxonate in order to examine the effect of isoproterenol on renal uric acid excretion.

Demartini (9) reported that epinephrine and norepinephrine decreased urine flow. $C_{V A}$ and $C_{U A} / C_{I n}$ in humans. In our preliminary experiment, epinephrine had a tendency to decrease $U_{v_{01}}$ and $U_{\mathrm{UA}}$; but norepinephrine was diuretic and slightly uricosuric in rats. The discrepancy between these observations is ascribed to variable

Table 2. Effect of isoproterenol on plasma uric acid in rats with ligated renal vessels

\begin{tabular}{llcl}
\hline Pretreatment & Drug & $N$ & $\begin{array}{c}\text { Plasma uric acid } \\
(\mathrm{mg} / \mathrm{dl})\end{array}$ \\
None & Saline & 4 & $1.86 \pm 0.45$ \\
& Isoproterenol & 4 & $2.99 \pm 0.80^{*}$ \\
Allopurinol & Saline & 4 & $0.69 \pm 0.15$ \\
& Isoproterenol & 4 & $0.62 \pm 0.09$ \\
\hline
\end{tabular}

Allopurinol $(100 \mathrm{mg} / \mathrm{kg}$. p.o.) was treated $60 \mathrm{~min}$ before ligation of renal vessels. Saline or isoproterenol $(50 \mu \mathrm{g} / \mathrm{kg}$, i.v.) was administered $10 \mathrm{~min}$ after the ligation. Data represent the mean \pm S.D. of the values at $30 \mathrm{~min}$ after drug administration. $*<<0.05$. significantly different from the control (saline control). 
effects of catecholamines depending on dosage and route of administration (10). Consequently, isoproterenol was expected to change renal uric acid excretion.

We observed that isoproterenol produced antidiuresis and hyperuricemia in oxonatenonloaded rats. These data agreed with those in the previous reports showing that isoproterenol is antidiuretic $(11,12)$ and hyperuricemic $(2,4)$. This drug first decreased. then increased, $U_{U_{A}}, C_{U_{A}}$ and $C_{U_{A}} / C_{I_{n}}$ i therefore, it seems likely that alteration of renal uric acid excretion plays an important role in regulating $P_{U_{A}}$. Propranolol induced mild diuresis and completely antagonized both the hyperuricemic effect and the renal effect of isoproterenol, suggesting that the effects of isoproterenol are related to betaadrenergic stimulation.

Although no increases in $C_{U A}$ and $C_{U A} / C_{I n}$ at $30 \mathrm{~min}$ after isoproterenol administration in oxonate-loaded rats were observed, other responses to isoproterenol were very similar to those obtained in oxonate-nonloaded rats. The decreases in $C_{\text {In }}$ shown in these two animal groups were less than those in

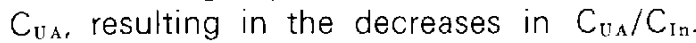
These results indicate that the inhibitory action of isoproterenol on renal uric acid excretion is due to both interference with glomerular tiltration and alteration of tubular transport of uric acid. Isoproterenol increases sodium reabsorption, leading to a decrease in renal sodium excretion (13). The reabsorption of sodium parallels that of uric acid (14). Therefore, the decrease in $C_{C_{\Lambda}} / C_{I_{n}}$ observed in the present study may reflect an increase in reabsorption of uric acid. Further studies are required to clarify this point.

We examined phenylephrine in oxonateloaded rats. As phenylephrine caused a transient increase in $U_{\mathrm{CA}}$ with diuresis, it is thought that if alpha-agonists such as phenylephrine and norepinephrine cause hyperuricemia $(4,15)$, the mechanism of the hyperuricemic action mediated by alphaadrenoceptors may involve only the stimulation of uric acid production.

Renal functions are closely related to blood pressure (16), and hypotensive action of isoproterenol is caused by stimulation of beta $_{2}$-adrenoceptors $(17,18)$. In the present study, the hypotensive effect of isoproterenol and the hypertensive effect (19) of phenylephrine were observed, and the changes in blood pressure were synchronized with those in $U_{U_{A}}$. These data point out the relationship between blood pressure and renal uric acid excretion, and they support our previous study in which the hyperuricemic action of isoproterenol was suggested to be related to beta ${ }_{2}$-adrenoceptors rather than to beta $_{1}$-adrenoceptors (15).

When the animals receiving the loading solution containing oxonate and uric acid were pretreated with allopurinol, isoproterenol caused mild hyperuricemia. This increase in $P_{U A}$ was probably due to the accumulation of uric acid in plasma induced by the depression of renal uric acid excretion. In addition, in rats which had lost renal functions as a result of ligature of renal vessels, the hyperuricemic effect of isoproterenol was completely inhibited by allopurinol.

In conclusion, the mechanisms of hyperuricemic action of isoproterenol involve not only the stimulation of uric acid production, but also the depression of renal uric acid excretion.

Acknowledgment: We thank Dr. S. Katagiri and Dr. M. Kagoshima for their helpful advice and Mr. H. Takimoto for his excellent technical assistance.

\section{References}

1 Yonetani, Y., Douzaki, T. and Ogawa, Y.: Epinephrine-induced hyperuricemia in expri. mental animals. Chem. Pharm. Bull. (Tokyo) 25, 441-447 (1977)

2 Sumi, T. and Umeda, Y.: Adrenergic regulation of the plasma levels of purine metabolites in the rat. Eur. J. Pharmacol. 46, 243-247 (1977)

3 Holmes, E.W., Jr.: Regulation of purine biosynthesis de novo. In Handbook of Experimental Pharmacology, Ed!ted by Kelly, W.N. and Weiner, I.M., Vol. 51, p. 21-41. Springer-Verlag, Berlin. Heidelberg and New York (1978)

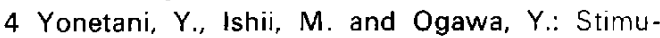
lation by catecholamine of purine catabolism in rats and chickens. Japan. J. Pharmacol. 29, $211-$ 221 (1979)

5 Johnson, W.J., Stavric, B. and Chartrand, A.: Uricase inhibition in the rat by s-triazines: An animal model for hyperuricemia and hyperuricosuria (33791). Proc. Soc. Exp. Biol. Med. $131,8-12$ (1969) 
6 Vurek, G.G. and Pegram, S.E.: Fluorometric method for the determination of nanogram quantities of inulin. Anal. Biochem. 16, 409-419 (1966)

7 Yonetani, Y. and Iwaki, K.: Effects of uricosuric drugs and diuretics on uric acid excretion in oxonate-treated rats. Japan. J. Pharmacol. 33, 947-954 (1983)

6 De Rougemont, D., Henchoz, M. and RochRamel, F.: Renal urate excretion at various plasma concentrations in the rat: a free-flow micropuncture study. Am. J. Physiol. 231, 387-391 (1976)

9 Demartini, F.E.: Hyperuricemia induced by drugs. Arthritis Rheum. 8. 823-827 (1965)

10 Osswald, $H$. and Greven, J.: Effects of adrenergic activators and inhibitors on kidney function. In Handbook of Experimental Pharmacology. Edited by Szekeres, L., Vol. 54/11, p. 243-288. Springer-Verlag. Berlin. Heidelberg and New York (1981)

11 Botting, R.M. and Lockett, M.F.: Threshold effect of subcutaneous adrenaline, noradrenaline and isoprenaline on water diuresis in rats. Arch. Int. Physiol. Biochim. 69, 36-45 (1961)

12 Yamazaki, N. and Monma, Y.: A study on the renal action of beta, - and beta $_{2}$-adrenoceptor in rats. Folia Pharmacol. Japon. 88, 271-277 (1986) (Abs. in English)

13 Greven, J. and Heidenreich, O.: A micropuncture study of the effect of isoprenaline on renal tubular fluid and electrolyte transport in the rat. Naunyn Schmiedebergs Arch. Pharmacol. 287, 117-128(1975)

14 Weinman, E.J., Eknoyan, G. and Suki, W.N.: The influence of the extracellular fluid volume on the tubular reabsorption of uric acid. J. Clin. Invest. 55, 283-291 (1975)

15 Sugino, $H$., Kagoshima, $M$, and Katagiri, $S$ : Effects of some drugs on plasma uric acid in rats: actions of catecholamines and $\beta$-blocking agents. Folia Pharmacol. Japon. 84, 293-301 (1984) (Abs. in English)

16 Guyton, A.C.: Textbook of Medical Physiology. Seventh edition, W.B. Saunders Company. Philadelphia, London, Toronto, Mexico City. Rio de Janeiro. Sydney. Tokyo and Hong Kong (1986)

17 Levy, B.: The adrenergic blocking activity of $\mathrm{N}$ tert.-butylmethoxamine (butoxamine). J. Pharmacol. Exp. Ther. 151, 413-422 (1966)

18 Shibouta, Y., Inada, Y., Terashita, Z., Nishikawa, $K$. and Kikuchi, S.: Antidiuresis induced by $\beta_{1}$ and $\beta_{2}$-adrenergic agonists in ethanol-anesthetized rats. Eur. J. Pharmacol. 47, 149-157 (1978)

19 Weiner, N.: Norepinephrine, epinephrine and the sympathomimetic amines. In The Pharmacological Basis of Therapeutics, Edited by Gilman, A.G., Goodman, L.S. and Gilman. A., p. 138-175. MacMillan Publishing Co. Inc., New York (1980) 